\title{
High-resolution 3D X-ray imaging of intracranial nitinol stents
}

\author{
Rudolph M. Snoeren • Michael Söderman • \\ Johannes N. Kroon • Ruben B. Roijers • \\ Peter H. N. de With • Drazenko Babic
}

Received: 17 November 2010 / Accepted: 26 January 2011 /Published online: 18 February 2011

(C) The Author(s) 2011. This article is published with open access at Springerlink.com

\begin{abstract}
Introduction To assess an optimized 3D imaging protocol for intracranial nitinol stents in 3D C-arm flat detector imaging. For this purpose, an image quality simulation and an in vitro study was carried out.

Methods Nitinol stents of various brands were placed inside an anthropomorphic head phantom, using iodine contrast. Experiments with objects were preceded by image quality and dose simulations. We varied X-ray imaging parameters in a commercially interventional X-ray system to set $3 \mathrm{D}$ image quality in the contrast-noise-sharpness space. Beam quality was varied to evaluate contrast of the stents while keeping absorbed dose below recommended values. Two detector formats were used, paired with an appropriate pixel size and X-ray focus size. Zoomed reconstructions were carried out and snapshot images acquired. High contrast spatial resolution was assessed with a CT phantom.

Results We found an optimal protocol for imaging intracranial nitinol stents. Contrast resolution was optimized for nickel-titanium-containing stents. A high spatial resolution larger than $2.1 \mathrm{lp} / \mathrm{mm}$ allows struts to be visualized. We
\end{abstract}

R. M. Snoeren $(\bowtie) \cdot$ P. H. N. de With

Faculty Electrical Engineering,

Signal Processing Systems group (SPS),

Eindhoven University of Technology (TU/e),

Laplace Building 028, Postbox 513, 5600MB Eindhoven,

The Netherlands

e-mail: r.m.snoeren@tue.nl

\section{Söderman}

Department of Neuroradiology, Karolinska University Hospital,

Stockholm, Sweden

J. N. Kroon $\cdot$ R. B. Roijers $\cdot$ D. Babic

Philips Healthcare,

Best, the Netherlands obtained images of stents of various brands and a representative set of images is shown. Independent of the make, struts can be imaged with virtually continuous strokes. Measured absorbed doses are shown to be lower than 50 mGy Computed Tomography Dose Index (CTDI). Conclusion By balancing the modulation transfer of the imaging components and tuning the high-contrast imaging capabilities, we have shown that thin nitinol stent wires can be reconstructed with high contrast-to-noise ratio and good detail, while keeping radiation doses within recommended values. Experimental results compare well with imaging simulations.

Keywords X-ray imaging · Cone beam 3D . Intracranial stents $\cdot$ Nitinol $\cdot$ Aneurysms

\section{Introduction}

The use of flat detectors allows display of vessel anatomy with submillimeter resolution and a high contrast-to-noise ratio (CNR). Three-dimensional (3D) cone beam imaging using a flat detector in a $\mathrm{C}$-arc system was adopted and eventually displayed an image quality approaching that of $\mathrm{CT}$ with respect to contrast resolution $[1,2]$. However, and despite the above improvement, 3D imaging of objects with high X-ray transparency and small detail may still be difficult [3]. Materials such as nitinol with its excellent biocompatibility and self deployment by shape memory [4] are widely used for intracranial stents, and generally yield good clinical results [5]. The usage of nitinol stents has become a common practice in the endovascular treatment as a coiling scaffold [6-8] to prevent wire herniation [9]. The visualization of nitinol stents in treatment of atherosclerotic stenoses $[10,11]$ is challenging and necessitates a 
highly developed X-ray imaging technique. Generally, in 2D clinical imaging protocols, only the stent end-markers made of tantalum or platinum can be recognized, but the stent body itself and struts are barely visible due to the low absorption of the constituents. To improve visualization, we need a high contrast resolution combined with high spatial resolution imaging. With 3D cone beam imaging based on flat detectors, both CNR and spatial resolution can be tailored such that fine detail rendition is sufficient to visualize the stent's struts. We describe a vascular imaging technique validated by an image quality assessment, using phantom objects with a variety of commercially available Nitinol stents. The purpose of this study is to visualize details of a stent, to support an improved analysis of its placement, by exploiting a joint optimization of all components of the imaging chain and the associated 3D vascular imaging platform.

\section{Materials and methods}

A proprietary SW package calculates the image quality in terms of signal flow through the entire imaging chain, using image quality descriptors like Modulation Transfer Function (MTF), impulse response, noise power spectrum, lowcontrast and high-contrast (spatial) resolution. Associated with these descriptors, the acquired dose can be calculated at any point in the physical chain, including a computed tomography dose index (CTDI) type of dose assessment for cone beam imaging. The analytical model also considers all the intra- and inter-component relations within the imaging system (also before and after detection). The quality description also incorporates an image quality degradation resulting from, e.g., system remnant blurring upon geometrical calibration and arc movement. The analytical model, comprehensively described by Kroon et al. [12, 13], allows us to vary the system parameters in X-ray generation, absorption, dose, and detection as well as in image processing quality, yielding accurate verified results.

A number of self-expandable nitinol stents (Table 1), as well as one steel-based stent for reference, were deployed in plastic (infuse) tubes with an inner diameter of $3.5 \mathrm{~mm}$ which were inserted in a channel of an anthropomorphic head phantom (CIRS, Norfolk, Virginia; model 603), placed in the system isocenter. The tubes were filled with a diluted contrast agent: $500 \mathrm{ml} \mathrm{H}_{2} \mathrm{O}$ and $50 \mathrm{ml}$ Visip 270 (GE), in order to produce a $550 \mathrm{HU}$ density mimicking a contrastfilled vessel. The remainder of the phantom channel was filled with diluted contrast agent: $5,000 \mathrm{ml} \mathrm{H}_{2} \mathrm{O}$ and $50 \mathrm{ml}$ Visip 270, producing 31-HU density. Another set of experiments was carried out by filling the tubes with $31-\mathrm{HU}$ diluted contrast agent and the remaining volume again with $31 \mathrm{HU}$, representing blood. Yet, another experiment was performed by inserting platinum coiling wires close to the stents, in an air-filled cavity in the phantom, enabling an assessment of streaking effects.

We used a Philips Xper ${ }^{\mathrm{TM}}$ vascular system equipped with a large $(30 \times 40 \mathrm{~cm})$ flat detector and a 3D workstation for producing the $3 \mathrm{D}$ rendered images and hosting the typical 3D image post-processing modules. Images were acquired with a standard 3D protocol (with 45-49 mGy CTDI dose) using a large detector zoom format in combination with a large tube focus, or in a zoomed detector format $(22-\mathrm{cm}$ imaging diagonal) and a small tube focus. A set of 620 images was acquired with a $30 \mathrm{fr} / \mathrm{s}$, resulting in a 20 -s scantime over a 200-degree arc-travel. CTDI was measured using a standard measurement protocol with a $16-\mathrm{cm}$ diameter polymethyl methacrylate (PMMA) cylinder, and dose was measured with the Unfors (Billdal, Sweden) Mult-O-Meter 601-PMS. The CTDI dose was kept below 50 mGy. We determined the values using the largest irradiation field while keeping the technique factors identical to those for
Table 1 An overview of the stent properties ${ }^{\mathrm{a}}$ The manufacturers did not supply
the dimensions

\begin{tabular}{|c|c|c|c|c|c|}
\hline Type & Manufacturer & Length (mm) & Diameter $(\mathrm{mm})$ & Strut cross-section $(\mu \mathrm{m})$ & Material \\
\hline Neuroform & $\begin{array}{l}\text { Boston Scientific } \\
\text { Natick, MA }\end{array}$ & 15 & 3 & $\mathrm{w} 68 * \mathrm{t} 66$ & Nitinol \\
\hline Enterprise & $\begin{array}{l}\text { Cordis } \\
\text { Bridgewater, NJ }\end{array}$ & 37 & 4.5 & $-^{\mathrm{a}}$ & Nitinol \\
\hline Solitaire & $\begin{array}{l}\text { ev3 } \\
\text { Plymouth, MN }\end{array}$ & 20 & 4 & $\varnothing 80$ & Nitinol \\
\hline Wingspan & $\begin{array}{l}\text { Boston Scientific } \\
\text { Natick, MA }\end{array}$ & 20 & 3.5 & $\mathrm{w} 68 * \mathrm{t} 73$ & Nitinol \\
\hline Silk & $\begin{array}{l}\text { Balt Extrusion } \\
\text { Montmorency, Fr }\end{array}$ & 25 & 3.5 & $-^{\mathrm{a}}$ & $\begin{array}{l}\text { Nitinol } \\
\text { Four Pt wires }\end{array}$ \\
\hline Leo+ & $\begin{array}{l}\text { Balt Extrusion } \\
\text { Montmorency, Fr }\end{array}$ & 25 & 2.5 & $-^{\mathrm{a}}$ & $\begin{array}{l}\text { Nitinol } \\
\text { Two Pt wires }\end{array}$ \\
\hline Pharos & $\begin{array}{l}\text { Micrus/Biotroniks } \\
\text { San Jose, CA }\end{array}$ & 25 & 2.75 & $\varnothing 60$ & $\begin{array}{l}\text { Steel } \\
\text { Cr-Co alloy }\end{array}$ \\
\hline
\end{tabular}


Table 2 Volume and voxel dimensions for the standard protocol and the high-resolution/high-contrast imaging protocol

\begin{tabular}{lccc}
\hline & $\begin{array}{l}\text { Reconstruction } \\
\text { zooming (\%) }\end{array}$ & $\begin{array}{l}\text { Volume }\left(256^{3} \text { voxel }\right. \\
\text { matrix })\left(\mathrm{mm}^{3}\right)\end{array}$ & $\begin{array}{l}\text { Voxel size } \\
(\mathrm{mm})\end{array}$ \\
\hline \multirow{2}{*}{ HIRES } & 50 & $52.8 \times 52.8 \times 52.8$ & 0.21 \\
& 33 & $34.4 \times 34.4 \times 34.4$ & 0.13 \\
\multirow{3}{*}{ Standard } & 33 & $82.7 \times 82.7 \times 63.9$ & 0.32 \\
& 17 & $42.6 \times 42.6 \times 32.9$ & 0.17 \\
\hline
\end{tabular}

the high-resolution case with its smaller field. The CTDI protocol would be meaningless for a smaller beam format, as it is not irradiating the complete cylinder with a diameter of $16 \mathrm{~cm}$, and thus not the peripheral probe positions. Spatial resolution was measured with a Catphan ${ }^{\circledR} 500$ phantom (The Phantom Factory, Salem NY). The resolution reading was limited by the phantom maximum of $2.1 \mathrm{lp} / \mathrm{mm}$. For the modeled limiting resolution analysis, a $4 \%$ modulation threshold was chosen. Object (physics governed) contrast was set by three tube voltages. This led us to the following protocols: standard 3D neuro protocol $(120 \mathrm{kV})$, intracranial stent protocol (ics, $80 \mathrm{kV}$ ) and ics high resolution (hires, $80 \mathrm{kV}$, zoomed detector format).

The reconstructions were carried out in a zoomed mode, i.e., a region of interest was chosen as a fraction of the maximum volume, determined by the detector size and the projection geometry. This volume is divided into the desired voxel matrix. The corresponding technical parameters are shown in Table 2, where linear reconstruction zoom factors of $50 \%$ and $33 \%$ for the HIRES protocol and $33 \%$ and $17 \%$ for the standard protocol are listed. A voxel matrix of $256^{3}$ was applied for all cases. The final images were rendered by maximum intensity projection with a slice thickness of $5.0 \mathrm{~mm}$, accommodating the stent's radial dimensions. The zoomed secondary reconstructions were carried out by panning the volume such that the relevant stent phantom portions were centered therein.

\section{Results}

The high contrast limiting resolution has been optimized by balancing the relevant MTF of the imaging components, taking noise transfer into account. Focus size, detector pixel size, and the reconstruction voxel size are matched such that the transfer contributions of each parameter are balanced, resulting in an optimal voxel size and voxel number. The nearest predefined volume is chosen such that it matches the size of the stent under test. A further reduction of the voxel size would increase the image noise, whereas an increase of the voxel size would result in a spatial resolution loss. The results on spatial limiting resolution are shown in Table 3. The true resolution of the high-resolution protocol is better than the maximum reading of the Catphan ${ }^{\mathrm{TM}} 500$ phantom and could thus not be read by this phantom. Deviations of $10-20 \%$ between measured and simulated values were found, which can be accounted for by the choice of the threshold modulation of $4 \%$. We optimized for the MTF and the inherent system impulse response, including the stent strut diameter of up to $0.08 \mathrm{~mm}$, as well as for contrast and noise. Since the basic materials of the stents are identical for all types (see Table 1), we decided to only use one stent type, namely a Wingspan nitinol stent. Only in case of the high-resolution/ high-contrast case, the imaging results of all stents are shown, including the Pharos reference stent.

\section{Reconstructed stent images inside a head phantom}

Figures 1, 2, 3, 4, and 5 show snapshots of reconstructed images in planes through the stent's axes and centered within the volume, where window settings on the work station were such that contrast and noise were comparable. For absolute stent dimensions, see Table 1. Figure 1 shows the results of the three protocols for $31 \mathrm{HU}$, where for reference, an optical image of the stent is shown also. The reconstruction scaling for the high- and standard resolution cases is found in Table 2. Figure 2 shows the images for the high contrast tube filling of $550 \mathrm{HU}$. Apart from the object contrast, the conditions are identical to those described for Fig. 1. The effect of varying the tube voltage is shown in Fig. 3, referring to a sequence of reconstructed images, displaying the image contrast for the infuse tubes filled with 31-HU contrast liquid. Figure 4 depicts the effect of streaking due to highly opaque platinum coils for $31-\mathrm{HU}$ filling. Finally, in Fig. 5, a number of stent types illustrate the
Table 3 Limiting high contrast spatial resolution as measured with a Catphan ${ }^{\circledR} 500$ phantom (The Phantom Factory) and simulated with the IQ analysis program

${ }^{\mathrm{a}}$ Catphan ${ }^{\circledR} 500$ phantom range limit

\begin{tabular}{llcc}
\hline Spatial resolution & Volume definition & Measured $(\mathrm{lp} / \mathrm{mm})$ & Simulated $(\mathrm{p} / \mathrm{mm})$ \\
\hline Standard & Non-zoomed reconstruction volume & 0.5 & 0.5 \\
& $33 \%$ reconstruction volume & 1.3 & 1.6 \\
& $17 \%$ reconstruction volume & 1.6 & 1.8 \\
HIRES & Non-zoomed reconstruction volume & 1.1 & 1.2 \\
& $50 \%$ reconstruction volume & 2.0 & 2.5 \\
& $33 \%$ reconstruction volume & $2.1^{\mathrm{a}}$ & 3.2 \\
\hline
\end{tabular}


Fig. 1 A Wingspan nitinol stent. A comparison between a the high-resolution/high-contrast case, b standard resolution/ high-contrast case, and $\mathbf{c}$ the standard resolution/standard contrast case. In all cases, an intra-tube contrast agent of $31 \mathrm{HU}$ was used. The relevant reconstruction (sub) volumes are denoted as $50 \%$ and $33 \%$ for the HIRES case. For the standard resolution, $17 \%$ and $33 \%$ images are shown. For comparison, a contrast inverted optical image is shown on the far left

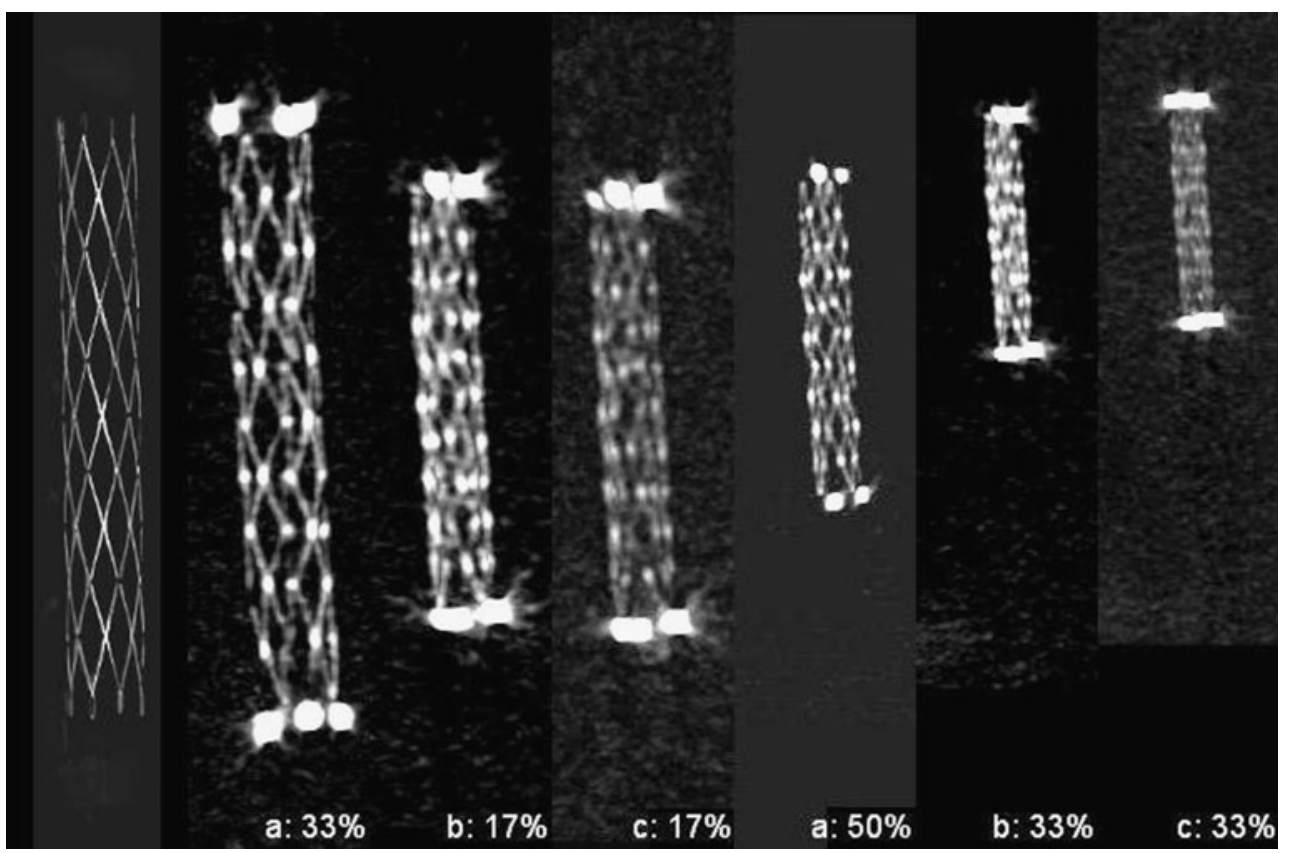

performance in the high-resolution/high-contrast mode, for again 31-HU contrast liquid. Figure 5 also shows example results of the more radiopaque material platinum in the Silk and Leo stents and the $\mathrm{Co}-\mathrm{Cr}$ steel alloy-based Pharos stent.

High contrast limiting spatial resolution

Measured and modeled limiting resolutions are summarized in Table 3. The results on measured resolution were found by reading the reconstructed images from a Catphan ${ }^{\mathrm{TM}} 500$ phantom in a transaxial plane.

\section{Dose CTDI}

By adjusting the technique factors, a CTDI-type weighted dose is kept at the desired low value of $50 \mathrm{mGy}$ for all protocols. The actual dose measurement and the analysis were carried out with a radiation field covering the $16-\mathrm{cm}$ CTDI phantom. Table 4 shows the measured and simulated values. The measured intersystem CTDI dose variability was $+/-0.5 \mathrm{mGy}$. We observed a systematic low error between measured and analytically modeled dose values.
Fig. 2 A Wingspan nitinol stent. A comparison between a the high-resolution/high-contrast case, b standard resolution/ high-contrast case, and $\mathbf{c}$ the standard resolution/standard contrast case. In all cases, an intra-tube high-density contrast agent of $550 \mathrm{HU}$ was applied. The relevant reconstruction (sub) volumes are denoted as $50 \%$ and $33 \%$ for the HIRES case. For the standard resolution, $17 \%$ and $33 \%$ images are shown

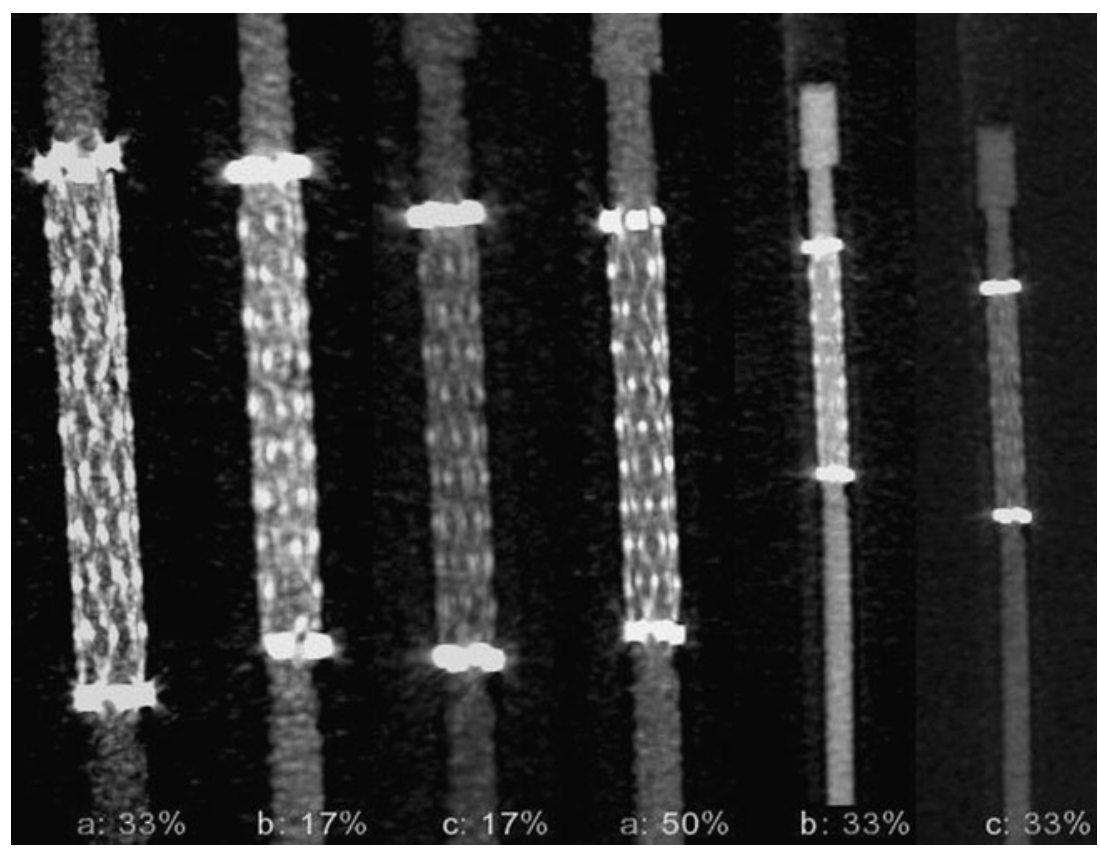


Fig. 3 A Wingspan nitinol stent. A comparison between the high-resolution cases for high-contrast $(80 \mathrm{kV})(\mathbf{a})$, intermediate contrast $(100 \mathrm{kV})$ (b), and standard contrast $(120 \mathrm{kV})(\mathbf{c})$. The $50 \%$ and $33 \%$ reconstruction volumes are shown. The applied intra-tube density was $31 \mathrm{HU}$

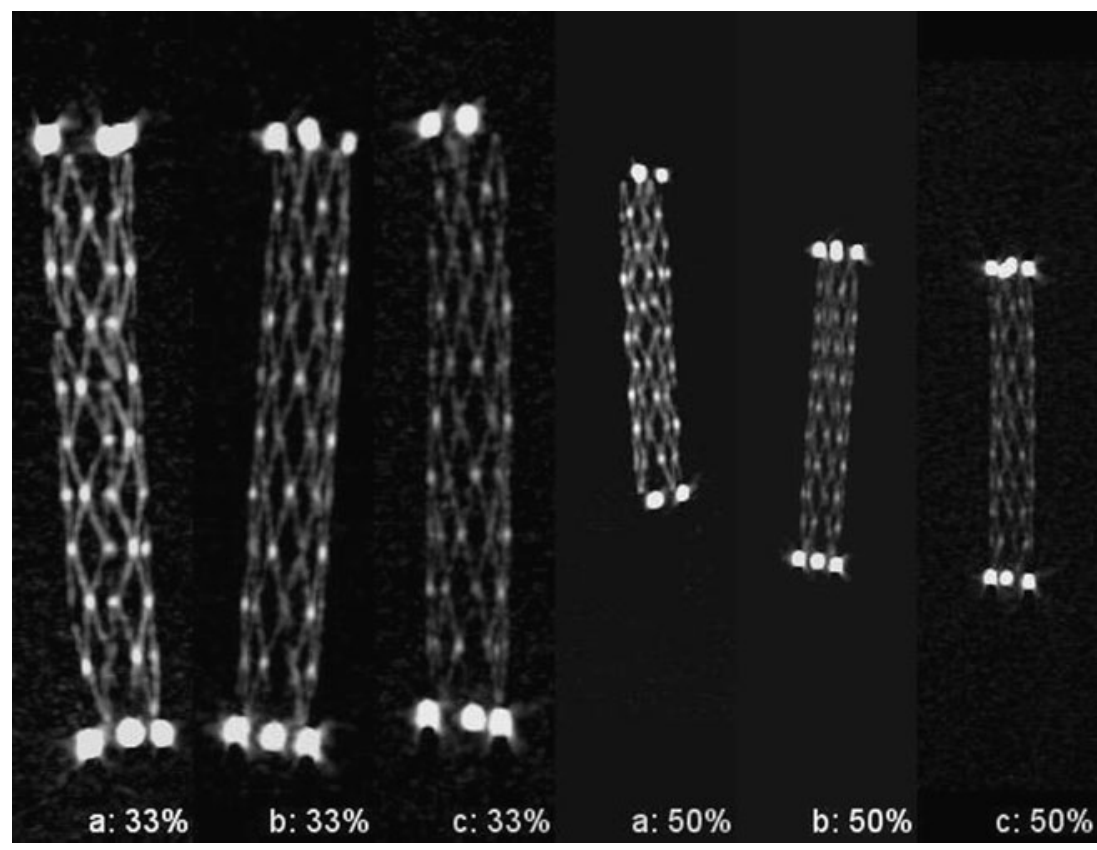

\section{Discussion}

Intracranial stenting became feasible as a routine treatment by the introduction of very flexible stents and have become a single treatment for a number of neurovascular conditions (Biondi et al. [14], Kurre et al. [15]). The stents are manufactured from a very thin material and most are self expanding, i.e., made from an alloy such as nitinol. However, most nitinol stents are difficult to visualize with fluoroscopy or C-arm cone beam CT. Visualization can be improved by increasing the density of the stent, more radiation, or enhanced image acquisition settings and processing. Increasing the density will add material and therefore unfavorably alters the stent characteristics and is therefore undesirable. An increase in dose will not be accepted (Struelens et al. [16]). Consequently, the visibility is preferably improved by further optimizing the imaging chain. To support and judge correct positioning, the nitinol stent should be visualized in its wall apposition, while conforming to varying diameters throughout the stent's length. To this end, it is advantageous to view details with virtually optical quality.

During the past 6 years, the image quality of CT-like reconstructions, using a $\mathrm{C}$-arm interventional $\mathrm{X}$-ray system equipped with a flat detector, became increasingly notable. High-resolution imaging, with submillimeter isotropic spatial resolution, outperformed digital radiography, fluoroscopy, and even conventional CT (Kamran et al. [17]). A continuous effort to enhance image quality enabled imaging of details with low absorption. Evidence on high-quality in vitro imaging of the proper deployment of nitinol stents, related to area coverage, kinking, prolapse, and flattening,
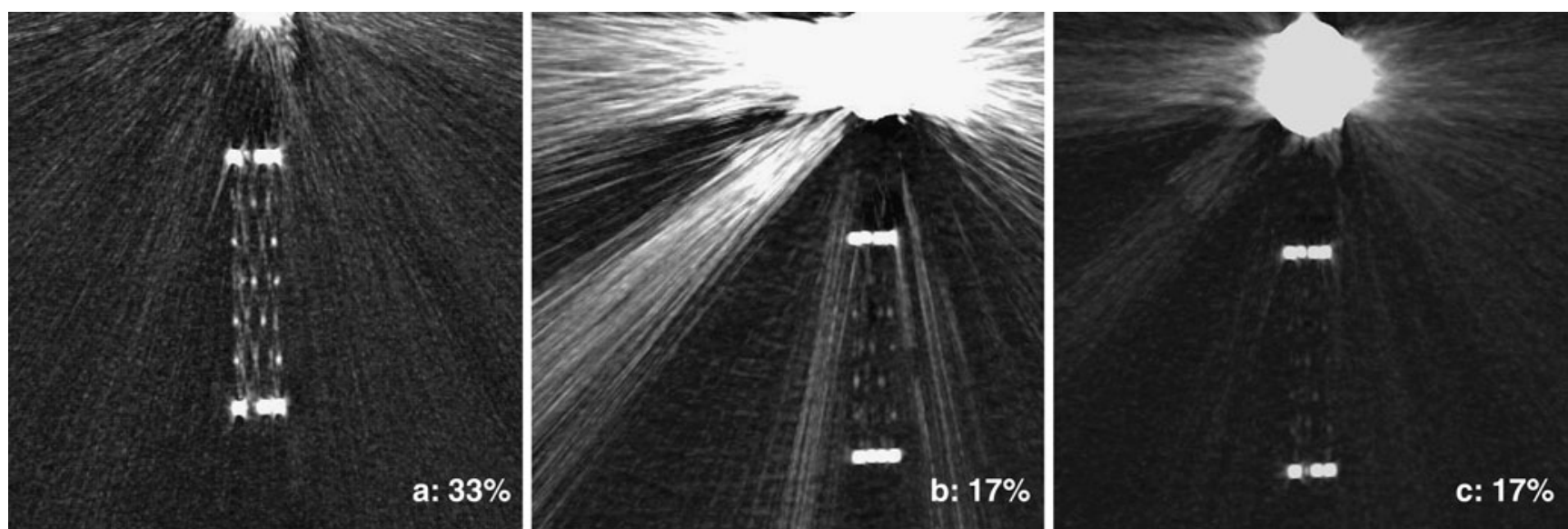

Fig. 4 Streak artifacts for Pt coils neighboring the Neuroform stent. a High-resolution/high-contrast, $\mathbf{b}$ standard resolution/high-contrast case, and c the standard resolution/standard contrast case 
Fig. 5 High-resolution/

high-contrast cases for $50 \%$ and $33 \%$ reconstruction

volumes and various stent types. The tubes were filled with 31-HU contrast. The Enterprise stent at $33 \%$ reconstruction volume was intentionally left out due to its large length not fitting this reduced volume. The steel-based Pharos stent was included for reference

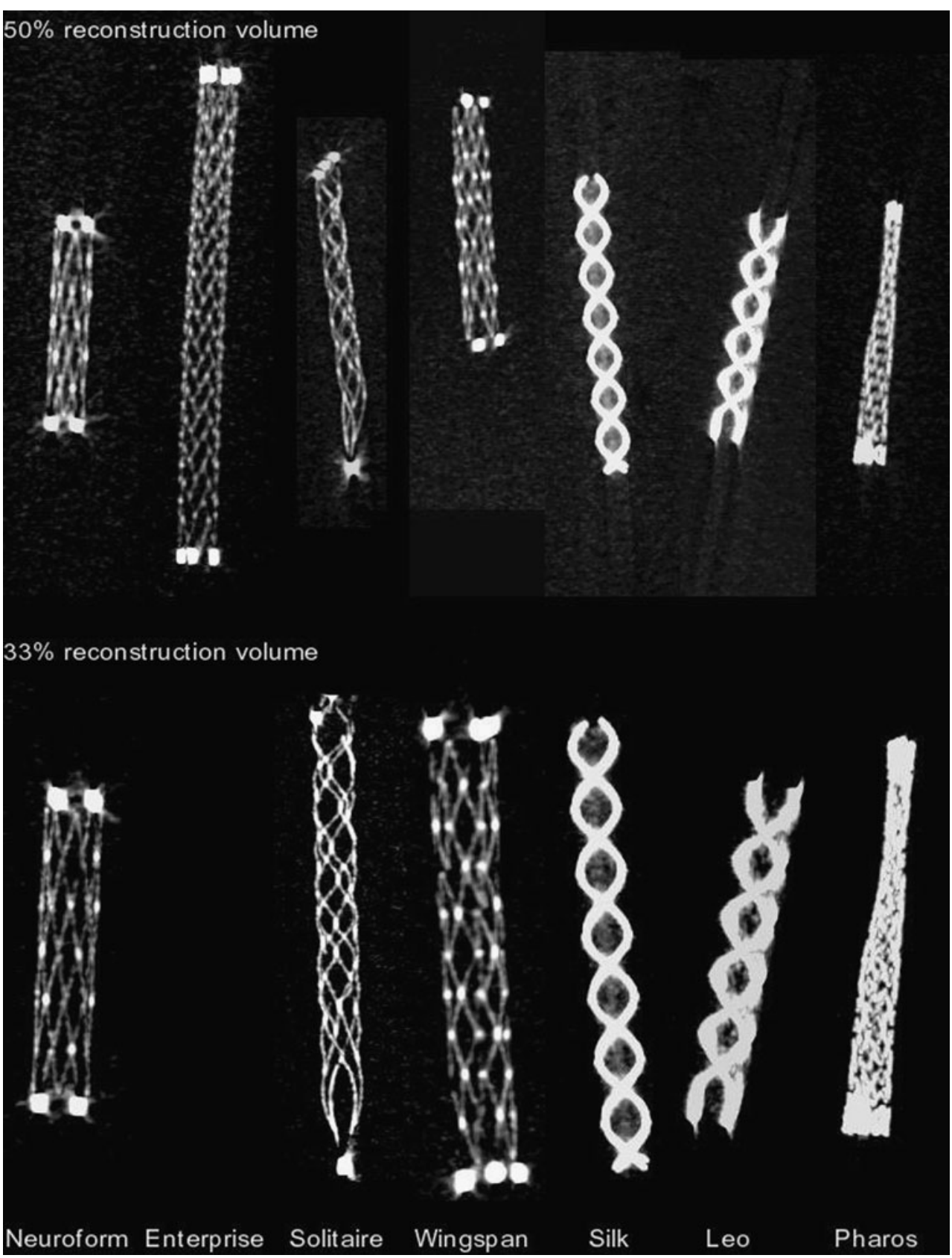

is given by Aurboonyawat [11], Ebrahimi [18] and Alvarado [19]. The stent conformity in curved vascular models and simulated aneurysm necks could be studied in detail. In a clinical setting, Benndorf [20] showed in vivo flat panel imaging of the Neuroform nitinol stent. Imagery

Table 4 Measured and simulated CTDI dose for the standard protocol and the high-resolution/high-contrast protocol

\begin{tabular}{lcc}
\hline CTDI & Measured dose (mGy) & Simulated dose (mGy) \\
\hline Standard & 45 & 43 \\
HIRES & 49 & 55 \\
\hline
\end{tabular}

of balloon mounted stents, based on a $\mathrm{Cr}-\mathrm{Co}$ steel alloy, with intravenous administration of contrast medium, was carried out by Buhk et al. [21]. The reconstructed images allowed an accurate assessment of the stented lumen. Recently, Patel et al. [22] demonstrated high-resolution and contrast-enhanced simultaneous imaging of intraarterial cerebrovascular stents and their host arteries.

In our study, a Wingspan stent was chosen as an arbitrary example object for an evaluation through reconstructed images. The image quality improvement is assessed by an evaluation of the reconstructed images. Clearly the spatial impulse response improved by introducing the HIRES protocol, as is shown in Fig. 1 for the 31-HU contrast-filled 
tube, where the CNR was improved as well by the increased object contrast. These two measures lead to an improved visibility of the stent's struts, when comparing the standard protocol (17\% zooming) with the results of the highresolution/high-contrast protocol (33\% zooming). The open cell structure of the stent is clearly discerned by virtually continuous strokes of the struts, so that its X-ray quality is approaching an optical quality. In Fig. 2, a comparison can be made for the 550-HU contrast filling. In this case, the contrast between struts and background is lower due to the denser filling of the tube, giving an accordingly reduced CNR. The sensitivity to object contrast can be readily viewed in Fig. 3, where the comparison is made for a varying tube voltage: the obvious increase in the CNR makes the stent more pronounced with respect to the (noisy) background and accounts for the choice of the lowest voltage, $80 \mathrm{kV}$, for the ICS and HIRES protocols. In Fig. 4, streak artifacts associated with high-density platinum coils are shown. Although the window settings are optimal for stent perception, details are still rendered with a sufficient contrast to artifact distance. Finally, Fig. 5 displays reconstructions of all stent types for the high-resolution/high-contrast case, showing the detail rendering of the protocol. As the cross-sections of the struts of the investigated types vary between 60 and $80 \mu \mathrm{m}$, the rendered contrasts vary accordingly, since the optimized system impulse response dominates for all cases. The Silk and Leo stents stand out more by high object contrast of the platinum auxiliary wires. In spite of the minimal strut diameter, the Pharos struts are rendered with the highest contrast. The $\mathrm{Co}-\mathrm{Cr}$ steel alloy accounts for an increased visibility due to its inherent higher absorption. The remaining stent types are comparable in their visualization quality, which is obvious, considering the similarity in dimensions and composition.

For all protocols under test, the maximum weighted CTDI dose of $50 \mathrm{mGy}$ is lower than the European guidelines, as according to EUR 16262 [23]. A dose of $60 \mathrm{mGy}$ is recommended for routine head examinations. Due to the limited anatomical coverage, by using a smaller irradiated field in the high-resolution case, the dose-length product is smaller as well. Moreover, we found that the difference between modeled and measured doses is sufficiently small.

\section{Conclusions}

We have optimized nitinol stent imaging, in the framework of a full-scale 3D imaging model for X-ray imaging. By balancing the sharpness transfer and noise transfer of the imaging components and tuning the contrast-rendering capabilities of a 3D X-ray imaging system, we showed that thin nitinol stent struts can be viewed with a high CNR and good detail rendering. While optimizing, the CTDI radiation doses were kept below recommended values.

The quality of 3D images, produced with optimum system settings, proved that independent of the type or manufacturer of the stents, the detail rendering is adequate to assess the post-deployment shape. The stent's struts can be imaged with virtually continuous strokes.

Conflict of interest We declare that we have no conflict of interest.

Open Access This article is distributed under the terms of the Creative Commons Attribution Noncommercial License which permits any noncommercial use, distribution, and reproduction in any medium, provided the original author(s) and source are credited.

\section{References}

1. Wallace MJ, Kuo MD, Glaiberman C et al (2008) Threedimensional C-arm cone-beam CT: applications in the interventional suite. J Vasc Interv Radiol 19:799-813

2. Orth RC, Wallace MJ, Kuo MD (2008) C-arm cone-beam CT: general principles and technical considerations for use in interventional radiology. J Vasc Interv Radiol 19:814-821

3. Söderman M, Babic D, Holmin S, Andersson T (2008) Brain imaging with a flat detector $\mathrm{C}$-arm technique and clinical interest of XperCT. Neuroradiology 50:863-868

4. Hoh DJ, Hoh BL, Amar AP et al (2009) Shape memory alloys: metallurgy, biocompatibility and biomechanics for neurosurgical applications. Neurosurgery 64:199-215

5. Sedat J, Chau Y, Mondot L et al (2009) Endovascular occlusion of intracranial wide-necked aneurysms with stenting (Neuroform) and coiling: mid term and long-term results. Neuroradiology 51:401-409

6. Weber W, Bendszus M, Kis B et al (2007) A new self-expanding nitinol stent (Enterprise) for the treatment of wide-necked intracranial aneurysms: initial clinical and angiographic results in 31 aneurysms. Neuroradiology 49:555-561

7. Peluso JPP, van Rooij WJ, Sluzewski M et al (2008) A new selfexpandable nitinol stent for the treatment of wide-neck aneurysms: initial clinical experience. AJNR Am J Neuroradiol 29:1405-1408

8. Wakhloo AK, Deleo MJ et al (2009) Advances in interventional neuroradiology. Stroke 40:305-312

9. Moret J, Cognard C, Weill A et al (1997) Reconstruction technique in the treatment of wide-neck intracranial aneurysms. J Neuroradiol 24(1):30-44

10. Bose A, Hartmann M, Henkes $H$ et al (2007) A novel, selfexpanding, nitinol stent in medically refractory intracranial atherosclerotic stenoses: the Wingspan study. Stroke 38:15311537

11. Thaweesak Aurboonyawat, Raphael Blanc, Paul Schmidt, Michel Piotin, Laurent Spelle, Amir Nakib, Jacques Moret (2011) An in vitro study of silk stent morphology. Neuroradiology. doi:10.1007/s00234-010-0784-4 
12. Kroon H, Schoumans N, Snoeren R (2006) Image quality simulation and verification of X-ray volume imaging systems. Proc SPIE 6142:614250

13. Kroon J (2005) 3-Dimensional rotational X-ray imaging, 3D-RX: image quality and patient dose simulation for optimization studies. Radiat Prot Dosim 114:341-349

14. Biondi A, Janardhan V, Katz JM, Salvaggio K, Riina HA, Gobin YP (2007) Neuroform stent-assisted coil embolization of wideneck intracranial aneurysms: strategies in stent deployment and midterm follow-up. Neurosurgery 61(3):460-469

15. Kurre W, Chapot R, du Mesnil de Rochemont R, Berkefeld J (2010) Intracranial stenting in atherosclerotic disease-recent results and challenges to face. Neuroradiology 52(7):633-644

16. Struelens L, Vanhavere F, Bosmans H, Van Loon R, Mol H (2005) Skin dose measurements on patients for diagnostic and interventional neuroradiology: a multicentre study. Radiat Prot Dosim 114 (1-3):143-146

17. Kamran M, Nagaraja S, Byrne J (2010) C-arm flat detector computed tomography: the technique and its applications in interventional neuro-radiology. Neuroradiology 52:319-327
18. Ebrahimi N, Claus B, Lee C, Biondi A, Benndorf G (2007) Stent conformity in curved vascular models with simulated aneurysm necks using flat panel CT: an in vitro study. Am J Neuroradiol 28:823-829

19. Alvarado M, Ebrahimi N, Benndorf G (2009) Study of conformability of the new LEO PLUS stent to a curved vascular model using flat-panel detector computed tomography (DynaCT). Neurosurgery $64: 130-134$

20. Benndorf G, Claus B, Strother C, Chang L, Klucznik R (2006) Increased cell opening and prolapse of struts of a neuroform stent in curved vasculature: value of angiographic computed tomography: technical case report. Neurosurgery 58:380-383

21. Buhk J, Lingor P, Knauth M (2008) Angiographic CT with intravenous administration of contrast medium is a noninvasive option for followup after intracranial stenting. Neuroradiology 50:349-354

22. Patel N, Gounis M, Wakhloo A et al (2011) Contrast-enhanced angiographic cone-beam $\mathrm{CT}$ of cerebrovascular stents: experimental optimization and clinical application. Am J Neuroradiol 32:137-144

23. Menzel HG, Scibilla H, Teunen D (2000) European guidelines on quality criteria for computed tomography. EUR 16262 EN 2000 